\section{SOLVING EXTREMUM PROBLEMS \\ WITH LINEAR FRACTIONAL OBJECTIVE FUNCTIONS ON THE COMBINATORIAL CONFIGURATION OF PERMUTATIONS UNDER MULTICRITERIALITY}

\author{
L. M. Koliechkina ${ }^{1 \dagger}$ and O. A. Dvirna $a^{1 \dagger}$
}

UDC 519.85

\begin{abstract}
The authors consider the extremum optimization problem with linear fractional objective functions on combinatorial configuration of permutations under multicriteria condition. Solution methods for linear fractional problems are analyzed to choose the approach to problem's solution. A solution technique based on graph theory is proposed. The algorithm of the modified coordinate method's subprogram with search optimization is described. It forms a set of points that satisfy additional constraints of the problem. The general solution algorithm without linearization of the objective function and it's block diagram are proposed. Examples of the algorithm are described.
\end{abstract}

Keywords: extremum problem, combinatorial configuration, linear fractional function, multicriteriality condition, modified coordinate method, search optimization.

\title{
INTRODUCTION
}

Multitasking in the majority of modern scientific problems that reflect practical activities of manufactures and organizations is related to vector optimization analyzed in $[1,5-10]$. Approaches to solution of vector problems are developed, for example, with the use of genetic algorithms [1], properties of sets on which the problem is solved [5-9], dialog procedures, various solution optimality criteria [10], etc. The form of objective functions and set of feasible solutions are of great importance in choosing the problem solution method.

In constructing models of economic and engineering processes related to planning company's activity, managing banking balance sheet items, estimating transportations, managing the activity of universities, etc., the need arises to optimize some relative parameter, which can be presented as a linear fractional objective function. If the domain of feasible values of such application-oriented problems has the properties of combinatorial configuration, then the extremum combinatorial problem analyzed in [2-9] is considered. This problem combined with vector optimization leads to the multicriteriality condition for the problem with linear fractional objective functions.

\section{STATEMENT OF THE EXTREMUM PROBLEM WITH LINEAR FRACTIONAL OBJECTIVE FUNCTIONS UNDER MULTICRITERIALITY}

Let us formulate the problem as follows: find $x^{*} \in D \subseteq X$ such that

$$
x^{*}=\arg \underset{x \in D \subseteq X}{\operatorname{extr}} F(x),
$$

${ }^{1}$ Poltava University of Economics and Trade, Poltava, Ukraine, †ludapl@ukr.net; ${ }^{\dagger}$ lenadvirna@gmail.com. Translated from Kibernetika i Sistemnyi Analiz, No. 4, July-August, 2017, pp. 113-123. Original article submitted January 27, 2017. 
where $F\left(f_{1}, f_{2}, \ldots, f_{n}\right)$ is a vector criterion that consists of linear fractional objective functions

$$
f_{i}=\operatorname{extr}_{x \in D \subseteq X} \frac{\sum_{j=1}^{m} c_{i j} x_{j}+c_{0}}{\sum_{j=1}^{m} d_{i j} x_{j}+d_{0}}, i \in N_{n}, j \in N_{m},
$$

under the condition $\sum_{j=1}^{m} d_{i j} x_{j}+d_{0} \neq 0, i \in N_{n}, j \in N_{m} ; D \subseteq X$ is the subset of feasible problem solutions, which is constructed from the system of additional linear constraint of the form

$$
a_{i t} x_{j} \leq b_{t}, \quad i \in N_{m}, t \in N_{k}
$$

$X$ is a combinatorial configuration;

$$
\text { extr } \in\{\min , \max \}
$$

is optimization direction; $n$ is the number of functions; $m$ is the number of variables; and $k$ is the number of problem constraints.

Problem (1)-(4) is vector one, with linear fractional objective functions on combinatorial set. To choose the method of problem solution, we will consider available approaches but adapted for multicriteria optimization problems.

\section{CHOOSING THE APPROACH TO THE SOLUTION OF COMBINATORIAL PROBLEM WITH LINEAR FRACTIONAL OBJECTIVE FUNCTION UNDER MULTICRITERIALITY}

The method of transformation of variables, which reduces the original problem to a linear one (linearization) is most often used to solve problems with linear fractional functions. The number of variables increases in this case, which transforms additional constraints of the problem. In case of combinatorial optimization, mapping of the given configuration is required. If multicriteriality condition occurs, the problem of transformation of variables becomes much more complicated since the denominators of objective functions are different, and standard formulas for linearization cannot be used for all the criteria simultaneously.

The method of objective function update is also applied. It provides periodic recalculation of local gradient of linear fractional function and reduces the linear fractional programming problem to a sequence of linear problems [10]. This method was developed for one-criterion optimization. As the number of variables increases and multicriteriality condition occurs, its computing complexity increases and modification becomes necessary.

The study [4] represents a solution technique for optimization problems with linear fractional function on the set of permutations, based on the combinatorial cutoff method. An advantage of this method is its practical efficiency for the specified class of problems as well as the use of linear fractional function in reducing vector problems to scalar ones. This method is applied to one-criterion optimization and employs the linearization method; therefore, in case of multicriteriality, it leads to the same problems as in the method of transformation of variables.

Thus, it is obvious that finding a universal algorithm to solve combinatorial vector linear fractional problems is a promising field for investigation. Multicriteriality combined with combinatorial properties complicates the problem, and specific approaches are necessary to solve models of such problems.

In the present paper, we propose to use the modified coordinate method applied earlier to linear problems [3, 6]. This approach does not need transformation of linear fractional criteria into linear ones since it deals immediately with the system of constraints. It allows finding only the combinatorial configuration points that satisfy problem conditions. The set of problem solutions is formed by comparing the values of optimality criterion at the found points of the given configuration that satisfy linear constraints of the problem.

The multicriteriality condition involves a number of difficulties since optimality criteria are often contradictory. When developing vector optimization methods, it is important to answer the question what solutions can be found as a result of its application. As a rule, the solutions belong to one of the following sets: 